\title{
Erratum to: The Perception and Cognition of Visual Space
}

\section{Erratum to: \\ P. Linton, The Perception and Cognition of Visual Space https://doi.org/10.1007/978-3-319-66293-0}

In the original version of the book, the post-publication corrections from author have been incorporated. The erratum book has been updated with the changes.

The updated online version of the book can be found at https://doi.org/10.1007/978-3-319-66293-0

(C) The Editor(s) (if applicable) and The Author(s) 2017 https://doi.org/10.1007/978-3-319-66293-0_5 